\title{
Distance Learning Classroom Using Virtual Harlem
}

\author{
Kyoung S. Park ${ }^{1}$, Jason Leigh ${ }^{1}$, Andrew E. Johnson ${ }^{1}$, Bryan Carter ${ }^{2}$, \\ Jennifer Brody ${ }^{3}$, and James Sosnoski ${ }^{3}$ \\ ${ }^{1}$ Electronic Visualization Laboratory at the University of Illinois at Chicago, \\ ${ }^{2}$ Department of English at the University of Illinois at Chicago, ${ }^{3}$ Department of \\ English at the Central Missouri State University \\ vh@evl.uic.edu
}

\begin{abstract}
Virtual Harlem is a virtual reality reconstruction of Harlem, New York, during the 1920s. It was designed to immerse students of the Harlem Renaissance directly in the historical context of the literature of that period. The goal of this prototype is to develop rich, interactive, and narrative learning experiences to augment classroom activities for students in the humanities. This paper describes a semester-long user study using Virtual Harlem in an English literature course and discusses our experiences of integrating virtual reality technology in the classroom.
\end{abstract}

\section{Introduction}

In the early 1900 s, particularly in the 1920 s, African-American literature, art, music, dance, and social commentary began to flourish in Harlem, a section of New York City. This African-American cultural movement became known as "The New Negro Movement" and later as "The Harlem Renaissance". The Harlem Renaissance transformed African-American identity and history, but it also transformed American culture in general. Never before had so many Americans read the thoughts of AfricanAmericans and embraced the African-American community's productions, expressions, and style.

Virtual Harlem is a collaborative virtual reality tour of Harlem in which participants can travel back 80 years to see historical figures, and hear speeches and music from that period. The Virtual Harlem project is an effort to create a learning environment that can enrich students understanding of the Harlem Renaissance. We believe this prototype enables students to become more than passive receptors of information, which is so common in many literature courses.

There have been many immersive virtual reality applications built and tested for education in the past. Many of these educational VR applications aimed to teach abstract concepts (in physics, mathematics, environmental science, etc) [6, 17, 18]. Some addressed the general knowledge acquisition and concept formation by experiencing in a first person point of view $[2,9]$. Some were based on a collaborative virtual environment where students learned materials while interacting with other students or teachers $[8,10]$. Although these works gave us useful insight in the use of VR in education, it did not quite explore how VR could be used in a regular classroom setting.

In this paper we describe a semester-long user study between the Central Missouri State University (CMSU) and the University of Illinois at Chicago (UIC) using Virtual 


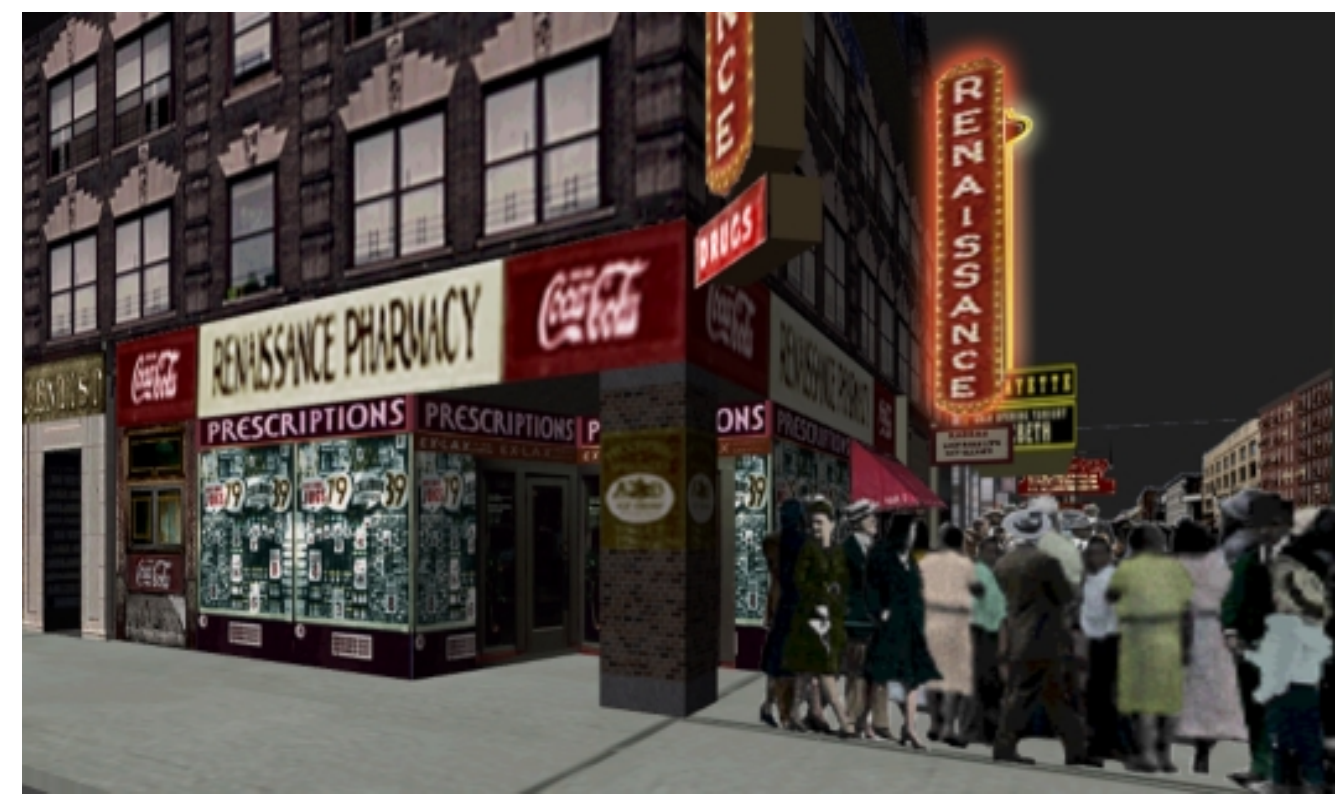

\section{Figure 1. Virtual Harlem allows students to walk through the streets of Harlem is in the 20s and learn more about the context of the literature they are studying.}

Harlem in an English literature course. The goal of the broader study was to explore how VR could be used to enhance traditional learning technologies such as books, videotapes, CDs etc, in the classroom. In the interest of brevity, this paper will focus only on the students' experiences with Virtual Harlem.

\section{Virtual Harlem}

Virtual Harlem was originally conceived in 1998 by Bryan Carter at the Central Missouri State University and the first prototype was created in collaboration with Bill Plummer at the Advanced Technology Center at the University of Missouri $[3,16]$. A year later, the University of Illinois at Chicago contributed to the project by translating the experience to a fully immersive environment - the CAVE® [5].

Designed as a supplement to a selection of literary works from the era, the Virtual Harlem prototype allows students to be immersed in the coursework. Students are able to walk down the streets of Harlem and see the shops, homes, theatres, churches, and businesses as well as the writers of that period. They can hear music in the Cotton Club, and political speeches of figures like Marcus Garvey, or thought-provoking poems by Langston Hughes.

Virtual Harlem has being written using a high-level VR toolkit called Yggdrasil (pronounced ig-dra-sil) [12,13]. Yggdrasil is a set of C++ classes built around SGI's IRIS Performer graphics library [15], CAVElib VR library [4] and the CAVERNsoft G2 networking toolkit [14]. Yggdrasil is designed to simplify the construction of behaviors for virtual objects using re-usable components; and on sharing the state of an environment through a distributed scene graph mechanism. Yggdrasil focuses on constructing dynamic, interactive, collaborative virtual worlds, so in addition to the basic graphical data as used in Performer, its scene graph nodes can have behaviors 


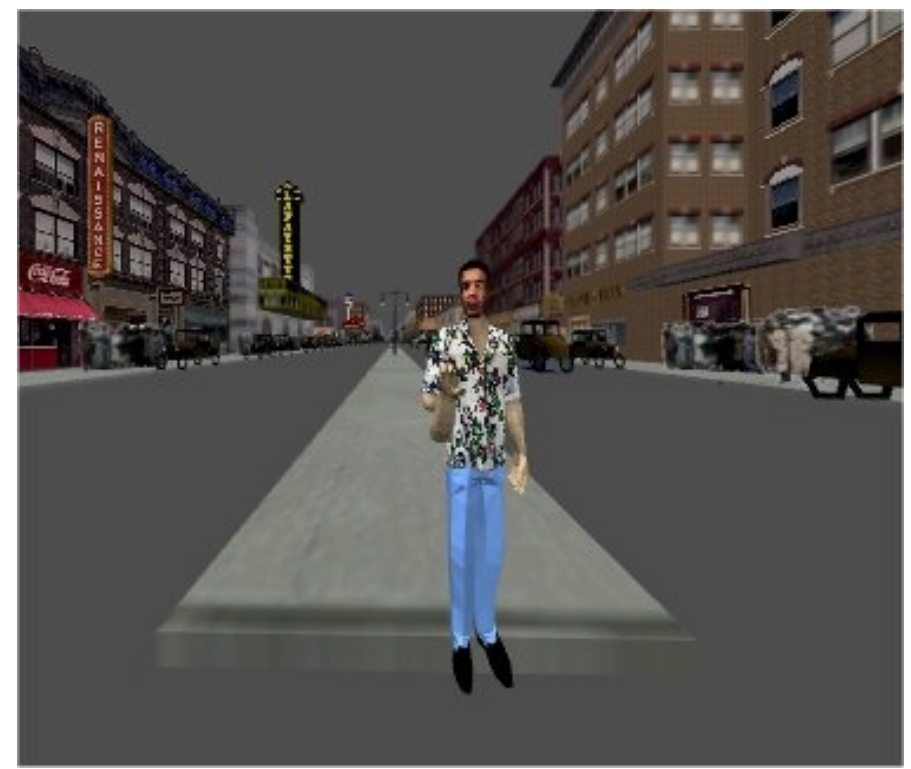

Figure 2. When an annotation is played back in Virtual Harlem as avatar
speaks and gestures just as the recorder of the annotation previously did.

attached to them. Individual node classes are compiled into dynamically shared objects (DSOs), so that they can be rapidly added to a world or modified. The system includes a number of pre-made classes that implement common capabilities in VR, such as audio playback, avatars, navigation controls, and triggers that detect when a user enters an area.

\section{Virtual Annotations}

All college students are familiar with the use of annotations. They use them to highlight important sections of a text, and make interpretive remarks to aid memory. We can similarly use annotations VR to highlight areas or events of interest that occur within the environment.

Virtual Harlem includes an annotation tool that allows students or instructors to leave annotations throughout the virtual space that can be retrieved by themselves or others in future visits. Virtual annotations are recordings in virtual reality where both the person's hand and head gestures, as well as their voice are captured. Since Virtual Harlem is a three-dimensional space the head and hand gestures allow the user to point toward landmarks in the space while speaking. When the annotations are played back an avatar appears to re-enact the annotation, as shown in Figure 2. Students were encouraged to form their own opinions on the things they saw and heard in the Harlem experience and then to leave annotations that other students could further comment on with their own annotations -hence creating a 'feed-back' loop. We hoped that this would further spur discussion and debate in the classroom or in the VR world.

The virtual annotation tool was developed as plug-in module for Yggdrasil. The recording engine is based on the VR-mail system [7]. It currently supports as the creation, play back and removal of annotations from within the virtual environment. All virtual annotations are saved in a persistent database server and loaded into a virtual 


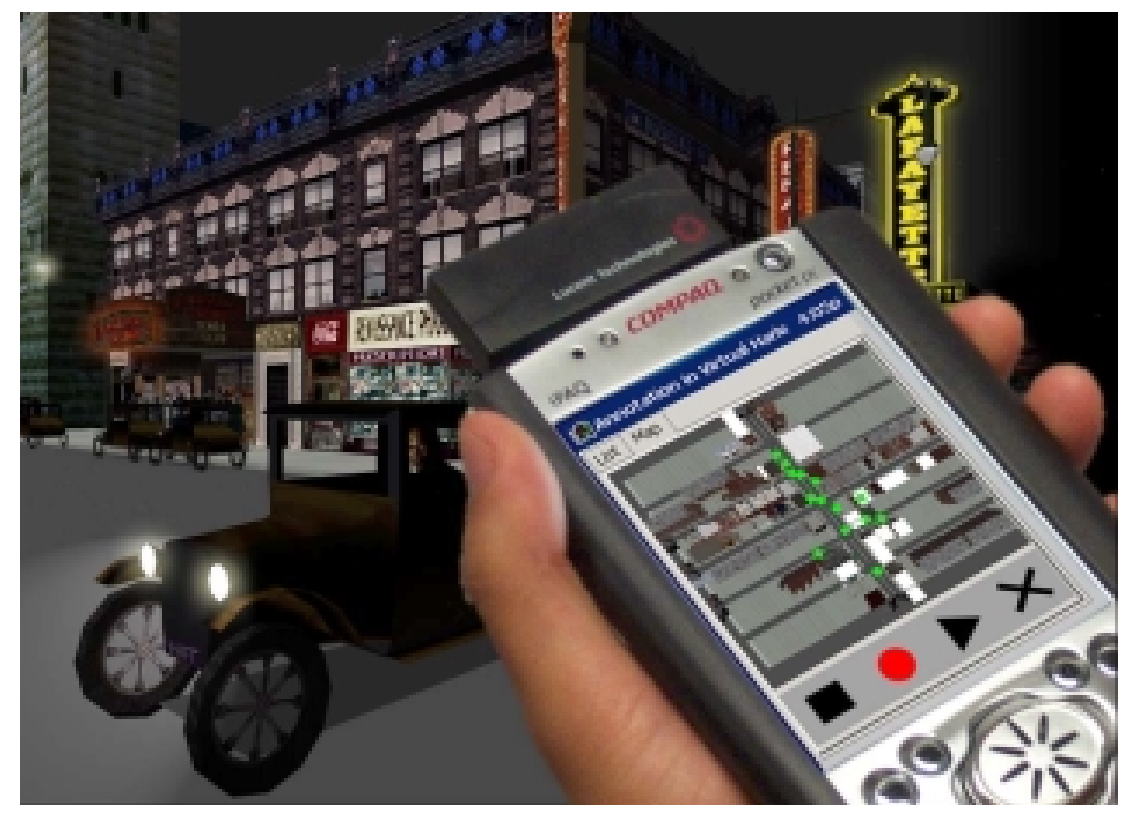

Figure 3. Using the PocketPC interface the user can see all of the annotations in the space. The list view shows who made the annotation and when it was made. The map view shows the locations of all the annotations.

environment by specifying a database file. The tool records the user's gesture and voice into separate files with headers that include the author's name, the annotation's name, and the location of the annotation in the virtual environment. Thus, it allows users to query the list of annotations by the author's name, region, or date.

In our current application, 3-dimensional avatar models are placed in the virtual environment as annotation markers to show the location and the author of the annotation. A user can activate the annotations by touching the marker. A user can stop a virtual annotation any time while it is playing. Furthermore the annotation will fade out as the user moves away from it. A user can record new annotations anywhere in the virtual environment, but only the owner of the annotation can remove a personal annotation.

In the collaborative session where several remote users are visiting Virtual Harlem at the same time, all users who are close to an annotation can hear it playing, but only the local user can hear an annotation as it is being recorded. The annotation is uploaded to the server as soon as it is completed. Annotations are deleted from the server when they are removed from the virtual environment.

Virtual Harlem, including the annotation tool, currently runs on both Silicon Graphics and Linux-based computers. Users with each machine type can collaborate and share annotations with each other. That is, virtual annotations that are recorded on SGI can also play back on Linux and vice versa.

\subsection{Using a Personal Digital Assistant in Virtual Harlem}

Virtual Harlem is a large space and the students can leave annotations anywhere. We needed another tool to find appropriate annotations. We may want to play back all the 
annotations done on a certain day, or made by a certain person, as well as at particular place. We have found, over the past decade of developing CAVE applications, that 3dimensional virtual menus are extremely difficult to operate for novice users. This is simply because it takes a tremendous amount of dexterity to be able to select menu items in 3D - especially when the frame rates are low. In Virtual Harlem, we chose to use a Personal Digital Assistant (PDA) as the Annotation Management Interface (AMIE). AMIE was developed for the IPAQ Pocket PC. In the CAVE a wireless LAN card provides tetherless connectivity between AMIE and the CAVE. Since the PDA is relatively light, it can be worn on a student's arm and activated with his/her finger.

AMIE provides two ways to access the annotations. Firstly it provides a list of all the annotations. Secondly, it provides a map of Harlem and the location of all the annotations (Figure 3). The list view allows students to access the annotations sorted by name, creator, or date/time. The map view provides feedback on where the student is currently standing in Harlem and their location relative to the annotation. Students skilled in navigation in VR can then travel there using the CAVE's wand or the AGAVE's joystick. Alternatively, less skilled students can achieve the same by clicking on one of annotations on the map. The system will then automatically shuttle the student to the annotation and then play it back to him/her. Furthermore the map view allows students to teleport to any point on the map.

\subsection{Providing Access to Annotations on the Web}

Recorded annotations are also distributed to the Web so that students can review the annotation while at home. The web site provides an interface similar to the one on AMIE to leverage the familiarity the students already have with AMIE. Hence, annotations are presented both on a map and in tabular form. Web-AMIE is written in a java-applet and is updated automatically when someone creates or deletes an annotation in the virtual environment. By clicking on one of the annotations, the audio of the annotation is played back.

\section{Distance Learning Classroom Using Virtual Harlem}

In the spring of 2001, the University of Illinois at Chicago (UIC) and the Central Missouri State University (CMSU) conducted a distance learning exercise, which bridged two English literature classrooms using a wide variety of collaboration technologies. The two courses on New York's Harlem Renaissance were taught simultaneously at CMSU (English4680: the African American Literature) by Bryan Carter and UIC (English350: the Harlem Renaissance) by Jennifer Brody. Both classes shared the same syllabus and reading materials. We wanted to investigate the use of common and exotic technologies in this collaboration. These included email, text, chat, online discussion boards, video conferencing and immersive virtual reality on both the CAVE and the AGAVE (Access Grid Augmented Virtual Environment) stereoscopic display wall.

AGAVE (Access Grid Augmented Virtual Environment) [11] provides passive polarized stereoscopic three-dimensional graphics using low-cost projectors and a Linux or Windows PC. This is the first time we have been able to bring our high-end visualization technology (typically costing between $\$ 150,000$ and $\$ 2,000,000$ ) to a cost low enough that it can be deployed in classrooms (around \$11000). AGAVE was designed to augment the Access Grid [1] to allow collaborators to immersively share three dimensional content, such as scientific and engineering data, in conjunction with 


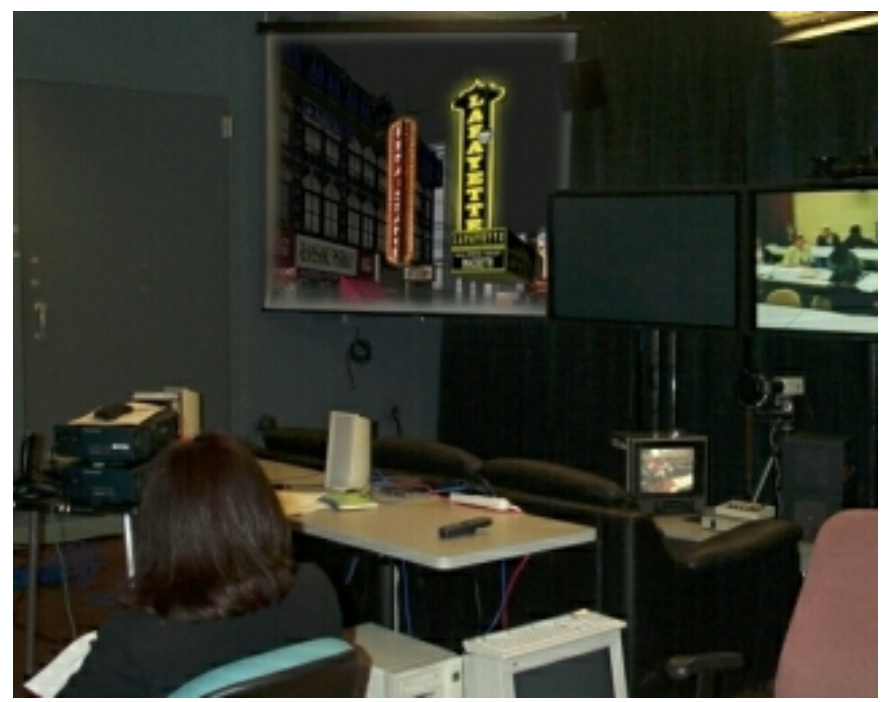

Figure 4. The AGAVE system allows us to bring Virtual Harlem into the classroom using passive polarized stereo visuals driven by a Linux PC. The screen on the right shows a video conference with the remote classroom.

their two dimensional Access Grid content - the Access Grid was designed primarily for multi-site video conferencing. With AGAVE, students wear inexpensive ( $\$ 0.30$ to \$12) 3D movie glasses to view the immersive content. If desired an additional 3D tracking system and pointing device can be incorporated to support $3 \mathrm{D}$ interaction. In this study we used a regular joystick. Figure 4 shows Virtual Harlem running on AGAVE. To the right of the AGAVE are plasma display screens of the Access Grid. On the rightmost screen is a video stream from the classroom at CMSU.

\subsection{Virtual Harlem Exploration and Experience}

In mid March students in both universities visited Virtual Harlem. Students were asked to explore Virtual Harlem and took notes on their experience as they normally would of anything else they study in their class. Students were organized into three groups. Half of UIC students were placed in the CAVE (16 students showed up in the class and all of them participated), the other half used the AGAVE (14 students), and all CMSU students watched the 10-minutes long pre-recorded walkthrough video (17 students). Jennifer's students were mostly senior-level undergraduate students. Bryan's students were in the graduate level. Most students were familiar with computer and Internet. However, none of them had prior experience with Virtual Harlem and CAVE or AGAVE technology.

The UIC students using the CAVE or AGAVE navigated around the Virtual Harlem space with the joystick for about $5 \sim 10$ minutes; there was no time limit. Usually $3 \sim 7$ students walked around the space in a group. On the other hand, CMSU students watched a video that contained the Virtual Harlem walkthrough with narrations. After exploring the space, they wrote general notes about what they saw and heard in the Virtual Harlem experience, what they would find interesting to see in the future and compare what they read about Harlem with what they experienced in the Virtual Harlem. 

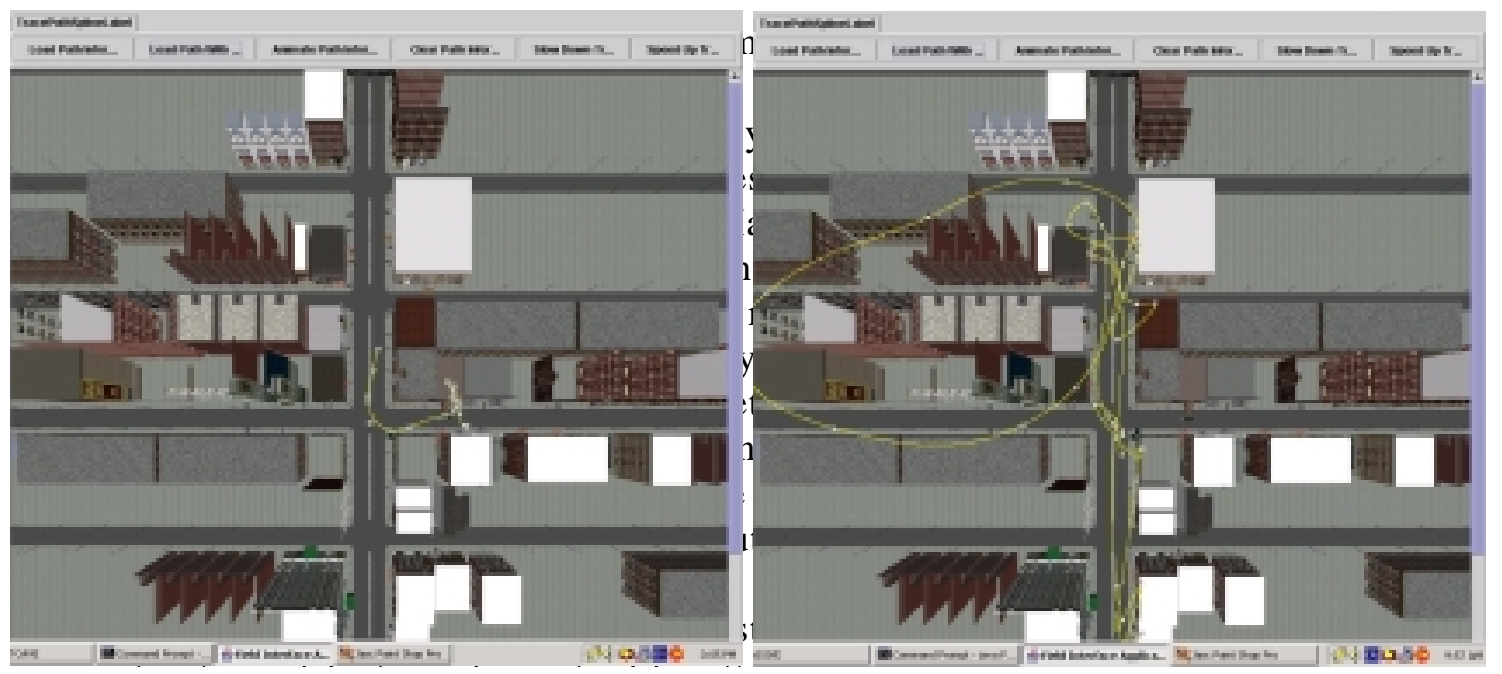

Figure 5. The left image is the trace patterns of students in the CAVE. The right image is the trace patterns of students using the AGAVE.

announce street names and point out places to go. Some also thought a hand held map would be an useful addition, to help navigate the space.

Students felt that the CAVE experience was better than the AGAVE experience. Students seemed to be more engaged in the CAVE; students in the CAVE were surrounded by the life- sized buildings and figures interacting with them whereas on the AGAVE students felt they were watching a 3D movie.

4.1.2. Results on trace patterns in the CAVE verses the AGAVE: During the Virtual Harlem exploration and note-taking session at UIC, all activities were recorded onto videotape and their paths through Harlem were logged. A separate log was created for each student/trip. Each log included a trace of student's navigation which would track how long they spent in each area of Virtual Harlem, how far they went, where they went, where they stood and looked around, etc. Interestingly, individual snapshots of the path traces showed quite different navigation patterns between the two VR systems. The students in the CAVE tended to stay stationary much of the time, the students using the AGAVE moved all over Harlem (Figure 5).

We believe this is primarily because the AGAVE did not provide a panoramic field of view, as in the CAVE, and hence students had to traverse each direction at a street intersection to orient themselves. For example, they were frequently searching for notable Harlem landmarks such as the Cotton Club or the Lafayette Theatre. In the CAVE they would simply turn their head in each direction to see if the landmark was in sight. Without a panoramic view they had to perform a 360-degree rotation, which can take about 30 seconds. Often from lack of patience, they did not perform a full rotation and hence missed the landmark. They would instead start-off in the first "promising" direction. This often got them lost and so they had to back tack often. 


\subsection{Virtual Harlem Annotations}

In mid April, UIC students were asked to create a short interactive narrative in Virtual Harlem using the VR annotation tool. They were asked to leave an annotation in Virtual Harlem that would enrich the experience for future students taking this course. The paper map was provided to help students find a location of interest in Harlem to leave an annotation. Each student would decid on the location for the annotation in Virtual Harlem, and then he or she would gave a brief 2 to 3 minute talk with the gestures. Students were organized into three groups: students who were previously in the CAVE were placed on the AGAVE, students who were previously on the AGAVE were placed in the CAVE; and CMSU students were only able to review the annotations through the web.

4.2.1. Observations on virtual annotations: Usually two students were paired-up to leave annotations in Virtual Harlem, and it took trials before they settled on the final annotation. Some students left more than one annotation. Some students grouped with other students to leave another annotation too. Students prepared their annotation topics before entering the Virtual Harlem. Unfortunately very few students left their own opinion on what they found in Harlem, as we had hoped. Instead, they read out portions of the textbook or some poetry.

Another important finding from this task was that most students did not wear 3D glasses while making the annotations because the glasses interfered with their reading of their textbook or notes inside the CAVE and AGAVE. The students did not wear the wireless headset microphone because they wanted to record several voices together and so they held the microphone in between them. In the CAVE the students could play back their annotations by moving over and 'touching' the annotation. Given the difficulty students had in navigating on the AGAVE we provided them with AMIE, which was very useful for playing back or removing an annotation to revise it.

Many students said they enjoyed leaving virtual annotations, particularly those in the CAVE. Some said putting together the annotations made it possible to utilize what they learned in class in a different and concrete way. It made them feel like they were a part of Virtual Harlem because they could leave their imprint behind through the annotation. On the other hand, some students were also concerned that any poorly made annotations would also become a permanent part of the environment.

\section{Conclusion and Future Work}

The Virtual Harlem Project is a collaborative effort between the University of Missouri, the Advanced Technology Center and the University of Illinois at Chicago, the Electronic Visualization Laboratory. This project is an effort to help students and scholars visualize what Harlem, New York, was like during the early part of the twentieth century. This paper described the Virtual Harlem project and our experiences in deploying it in an English literature course.

In this study we investigated design issues of integrating virtual reality technology in a college-level classroom. One of the major benefits that we expected from using VR in this literature course was to do with historiography - or more broadly "entering history". We believe that Virtual Harlem helped the students to think more about their complicity as readers of the environment, always already virtual, known as the "Renaissance." Through this experience, students were more engaged in classroom activities, e.g. more debates were spurred in the class after the VR experience. 
Overall students wanted more exposure to the technologies than what we provided in this study. The results on this study suggested that we should bring the technology to the classroom instead of the classroom to it; incorporate it more into the regular class work; and make it more available and inclusive to everyone. Unfortunately the study does not as yet yield a concrete guideline for how to integrate VR in the classroom. This is certainly one of the important goals in the future. However, this preliminary study showed the potential of using VR as a complimentary media to regular classroom media.

In the future we would like to encourage students to be content providers in Virtual Harlem. We would like to encourage them to imagine that they are a writer or artist in the 1920s in Harlem, and compose a poem, political speech, music, painting or photo of their own and place it in Virtual Harlem for others to see. Or if they have been to the real Harlem ask them to add a commentary on where Harlem is today in contrast to what they think it was like back them. Also, we would like to encourage students to leave more virtual annotations in Virtual Harlem to create a feedback loop of discussions within a virtual environment or in the classroom. Finally, we believe the greatest potential of Virtual Harlem, and VR for cultural heritage in general, is the re-enactment of historically controversial scenarios. Scholars can use this technology to reconstruct historical events from multiple interpretations so that they can be compared side-by-side.

\section{Acknowledgements}

We would like to thank all the students for serving as volunteers in this study. In particular we would like to thank Steve Jones, Tim Portlock, Yongjoo Cho, Eric He, Michael Lewis, Naveen Krishnaprasad, Shalini Venkataraman, and Janet Kim for setting up the system and helping us process the experiment. We would also like to thank Seung Kang, Natt Mintrask, and Joseph Tremonti for providing audio/visual supports.

The virtual reality research, collaborations, and outreach programs at the Electronic Visualization Laboratory (EVL) at the University of Illinois at Chicago are made possible by major funding from the National Science Foundation (NSF), awards EIA9802090, EIA-9871058, ANI-9980480, ANI-9730202, and ACI-9418068, as well as NSF Partnerships for Advanced Computational Infrastructure (PACI) cooperative agreement ACI-9619019 to the National Computational Science Alliance. EVL also receives major funding from the US Department of Energy (DOE), awards 99ER25388 and 99ER25405, as well as support from the DOE's Accelerated Strategic Computing Initiative (ASCI) Data and Visualization Corridor program. In addition, EVL receives funding from Pacific Interface on behalf of NTT Optical Network Systems Laboratory in Japan and Microsoft Corporation.

\section{References}

[1] Access Grid web page. http://www.accessgrid.org/

[2] Allison, D., Wills, B., Bowman, D., Wineman, J., Hodges, L. F., "The Virtual Reality Gorilla Exhibit", IEEE Computer Graphics and Applications, November/December 1997, pages 30-38.

[3] Carter, B., "Virtual Harlem”, SIGGRAPH '99 Conference Abstracts and Applications, 1999, pp. 103.

[4] CAVElib. http://www.vrco.com/

[5] Cruz-Neira, C., Sandin, D. J., DeFanti, T. A., Kenyon, R. V. and Hart, J. C., "The Cave Automatic Virtual Environment," Communications of the ACM, 35(2):64-72, June 1992.

[6] Dede, C., Salzman, M. C., Loftin, R. B., "ScienceSpace: Virtual Realities for Learning Complex and Abstract Scientific Concepts", 1997, http://www.vetl.uh.edu/ScienceSpace/absvir.html 
[7] Imai, T., Qui, Z., Behara, S., Tachi, S., Aoyama, T., Johnson, A., Leigh, J., "Overcoming Time-Zone Differences and Time Management Problem with Tele-Immersion", In the proceedings of INET 2000, July 18-21, 2000, Yokohama, Japan, CDROM.

[8] Jackson, R.L., Fagan, E., "Collaboration and Learning within Immersive Virtual Reality", In the proceedings of ACM CVE 2000, San Francisco, CA, September 10-12, 2000, pp. 83-92.

[9] Johnson, A., Moher, T., Ohlsson, S., Gillingham, M., “The Round Earth Project: Collaborative VR for Conceptual Learning", IEEE Computer Graphics and Applications, 19(6), November / December, 1999, pp. 60-69.

[10] Johnson, A., Roussos, M., Leigh, J., Barnes, C., Vasilakis, C., Moher, T., "The NICE Project: Learning Together in a Virtual World", In the proceedings of VRAIS '98, Atlanta, Georgia, Mar 14-18, 1998, pp. 176183.

[11]Leigh, J., Dawe, G., Talandis, J., He. E., Venkataraman, S., Ge, J., Sandin, D., DeFanti, T. A., “AGAVE : Access Grid Augmented Virtual Environment", In the proceedings of AccessGrid Retreat, Argonne, Illinois, Jan, 2001. (Jan 16, 2001)

[12] Pape, D., Composing Networked Virtual Environments, Ph.D. Dissertation, University of Illinois at Chicago, 2001.

[13] Pape, D., Anstey, J., Carter, B., Leigh, J., Roussou, M., Portlock, T., "Virtual Heritage at iGrid 2000", In the proceedings of INET 2001, Stockholm, Sweden, 5-8 June 2001.

[14] Park, K., Cho, Y., Krishnaparasad, N., Scharver, C., Lewis, M., Leigh, J., Johnson, A., "CAVERNsoft G2: a toolkit for high performance tele-immersive collaboration," In the proceedings of the ACM Symposium on Virtual Reality Software and Technology 2000, Oct22-25, 2000, Seoul, Korea, pp. 8-15.

[15] Rohlf, J., Helman, J., "IRIS Performer: A High Performance Multiprocessing Toolkit for Real-Time 3D Graphics”, In the proceedings of SIGGRAPH ‘94 Computer Graphics Conference, 1994, pp. 381-395.

[16] Virtual Harlem at Advanced Technology Center. http://www.atc.missouri.edu/virtualharlem/

[17] Winn, W., "The virtual reality roving vehicle project", T.H.E. Journal: Technology Horizons in Education Journal, 23(5), 1995, pp. 70-74.

[18] Winn, W., E., Bricken, W., "Designing Virtual Worlds for Use in Mathematics Education: The Example of Experiential Algebra”, Educational Technology, 32(12), 1992, pp. 12-19. 